\title{
All-normal-dispersion fiber lasers for frequency metrology
}

\author{
Çağrı Şenel ${ }^{1}$, F. Ömer Ilday ${ }^{1}$, Oğuzhan Kara ${ }^{2}$, Cengiz Birlikseven $^{3}$, Cihangir Erdoğan $^{3}$, Ramiz Hamid $^{3}$ \\ ${ }^{l}$ Department of Physics, Bilkent University, 06800, Ankara, Turkey \\ ${ }^{2}$ Department of Physics Engineering, Hacettepe University, 06532, Ankara, Turkey \\ ${ }^{3}$ TÜBİTAK, Ulusal Metroloji Enstitüsü (UME), 41470, Kocaeli, Turkey
}

\begin{abstract}
Development of an all-normal-dispersion Yb-doped fiber laser-based frequency comb is reported. Repetition-frequency stabilization to the cesium standard, amplitude and phase noise measurements indicate lownoise performance.

(C)2011 Optical Society of America

OCIS codes: (320.0320) Ultrafast optics; (060.3510) Lasers, fiber; (120.3940) Metrology; (140.3425) Laser stabilization; (140.3538) Lasers pulsed; (320.7090) Ultrafast Lasers; (320.6629) Supercontinuum generation
\end{abstract}

Mode-locked fiber lasers have emerged as very attractive sources for frequency metrology with their superior long-term stability and compact size [1]. Most fiber frequency combs are based on Er-doped fiber lasers, whereas Yb-doped fiber lasers centered at $1 \mu \mathrm{m}$ offer higher powers and their proximity of their central wavelength to the most commonly used optical reference lines, such as $\mathrm{I}_{2}, \mathrm{Rb}, \mathrm{Cs}$ are among their attractive features. There are few reports on frequency combs in this wavelength range [2]. All-normal dispersion (ANDi) fiber lasers have found broad usage due to their simplicity, compactness and higher pulse energies [3]. There is strong motivation to evaluate their suitability for frequency comb generation. The first work to address this issue has reported relatively poor noise performance, suggesting that they may not be well suited for this application [4]. This is an important claim that needs to be verified and the reasons for any poor performance should be understood.

Here, we report on the development of a frequency comb based on an Yb-doped ANDi-type fiber laser. We place particular emphasis on its long-term stability and transportability. Part of the experimental setup is shown in Fig. 1. The repetition rate of this oscillator has been locked to a frequency synthesizer, disciplined by a Cs-atomic clock. The repetition rate of the laser is $108.33 \mathrm{MHz}$. The short and long-term stability of the laser is characterized by relative intensity noise (RIN), single-side band RF phase noise and Allan variance measurements. RIN of the laser is measured as $0.072 \%$ within a bandwidth from $1 \mathrm{~Hz}$ to $250 \mathrm{kHz}$ (Fig. 1(b)). Phase noise of the laser is measured at $1.3 \mathrm{GHz}$ using direct photodetection. RMS timing jitter is measured to be $41 \mathrm{fs}$ from $1 \mathrm{kHz}$ to 30 MHz. The measurement is limited by excess noise from the measurement setup and should be considered as an upper limit to the actual phase noise. The RF spectrum shows 100-dB suppression of the sidebands (Fig. 2(a)).

Repetition-rate locking of the ANDi laser to the Cs clock is accomplished with a phase-locked loop (PLL). The $10-\mathrm{MHz}$ reference signal from the Cs atomic clock is up-converted to $1.3 \mathrm{GHz}$ through a DRO. Upon locking the laser to the Cs reference, the long-term stability performance fiber laser is characterized using Allan deviation using a time interval and frequency counter, which is externally triggered from the Cs standard. Instead of direct frequency count, time interval measurement method is preferred for improved fidelity. The frequency stability of two similar Cs atomic clocks was also measured using a short cable in the same room, revealing that white noise is dominant as expected and long-term stability is around $2 \times 10^{-14}$ (Fig. 2(b)). The long-term stability of the laser in both free-running and Cs-locked mode is given in Fig. 2(b). For free-running operation, repetition rate stability is dominated by drift noise. When locked to the Cs atomic clock, laser reaches a stability of $4 \times 10^{-14}$ after 65536 seconds of averaging time, confirming that flicker and drift noise do not affect its stability in the long term.

For carrier-envelope phase stabilization, an octave-spanning frequency comb is generated after amplification in a cladding-pumped $\mathrm{Yb}$-doped fiber amplifier, similar to the setup described in [5]. The amplified pulses are compressed to sub-300 fs at an average power of $2 \mathrm{~W}$. The beam is coupled into $60-\mathrm{cm}$-long nonlinear photonic crystal fiber (PCF) with zero dispersion at $1050 \mathrm{~nm}$ (Fig. 3(a)). An f-2f interferometer (using second-harmonic of the components at $1400 \mathrm{~nm}$ in a BBO crystal and light at $700 \mathrm{~nm}$ ) for the measurement and stabilization of $\mathrm{f}_{\text {ceo }}$ is currently under construction.

We intend to utilize the comb system to compare with single-frequency lasers locked to optical references in the visible and NIR range. For these experiments, it is necessary to have a beat signal with SNR greater than $30 \mathrm{~dB}$ to be able to realiably measure the beat frequency using a frequency counter. A secondary goal is to provide an independent measurement of the optical phase noise of the fiber comb. We performed a beat experiment with Nd:YAG laser at UME. The beam from the Nd:YAG laser and the amplified ANDi laser are overlapped on a beamsplitter and the resulting heterodyne beat signal is detected and measured (Fig. 3(b)). Resulting beat signal has a 3-dB bandwidth smaller than $30 \mathrm{kHz}$. During this measurement, the fiber laser was not repetition-rate locked and the width of this beat signal is consistent with the measured RF phase noise of the fiber laser. The error signal obtained by using the beat signal was sent to PZT of Nd:YAG laser for phase locking to ANDi laser and a lock is reached with an uncertainty of $<1 \mathrm{kHz}$ (Fig. 3(b)). Allan deviation after 1 second is $393 \mathrm{~Hz}$ and it reaches value of $82 \mathrm{~Hz}$ after 256 seconds. The experiment for the precise registration of absorption spectrum of $\mathrm{R}(56) 32-0-\mathrm{a} 10$ line of iodine molecular gas is on progress. 

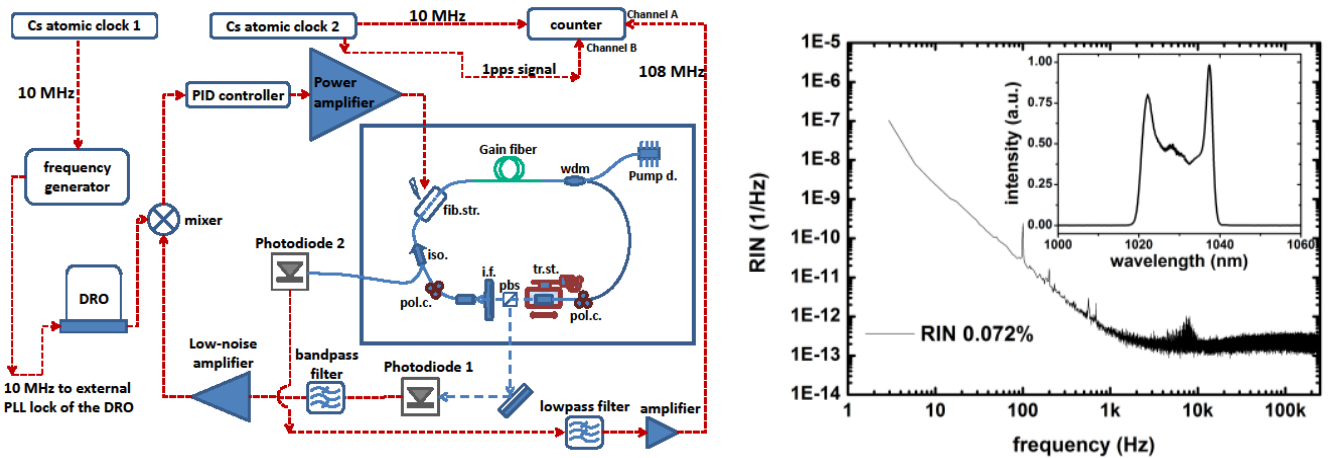

Fig. 1 (a) Overview of the experimental setup including schematic of the ANDi fiber laser and the PLL system. (b) Measured relative intensity noise (RIN) of the Yb-doped fiber laser, inset: optical spectrum of the fiber laser.
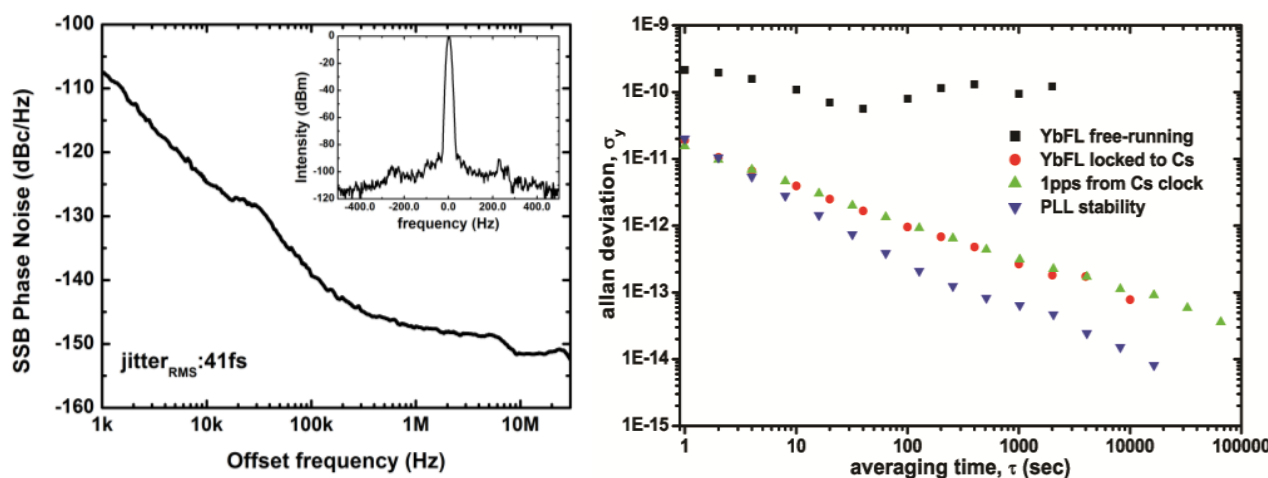

Fig. 2 (a) Single side band phase noise of the laser, inset: RF spectrum of the repetition frequency of the laser. (b) Allan deviation measurements of free-running YbFL, 1 pps signal from Cs atomic clock, Cs-locked YbFL and the PLL.
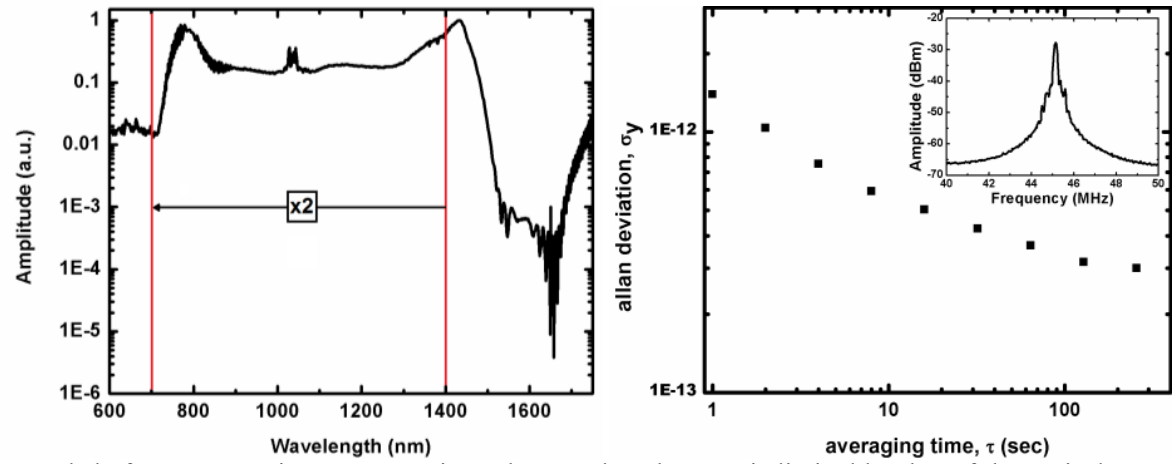

Fig. 3. (a) Spectrum recorded after supercontinuum generation. The wavelength range is limited by that of the optical spectrum analyzer. (b) Allan deviation measurement of beat signal after Nd:YAG phase locked to YbFL, inset: beat signal

In conclusion, an all-normal dispersion fiber laser has been repetition rate locked to a Cs atomic clock, inhereting its stability. The short-term stability of the laser is characterized via RIN and phase noise measurements, both of which indicate performance meeting or exceeding the performance with other types of fiber lasers. An octave-spanning supercontinuum has been stably generated, which will be used to stabilize the carrierenvelope offset phase. The long-term stability of the laser is excellent and surpasses the other fiber lasers we have so far tested: During the Allan deviation measurements lasting about one week, the laser has maintained modelocked operation, as well as repetition rate lock to the Cs reference without interruption, even though it was placed on an ordinary table, without any vibration isolation.

[1] N. R. Newbury, and W. C. Swann, "Low-noise fiber-laser frequency combs (Invited)," JOSA B 24, 1756 (2007) and references therein.

[2] T. R. Schibli, I. Hartl, D. C. Yost, M. J. Martin, A. Marcinkevicius, M. E. Fermann, and J. Ye, "Optical frequency comb with submillihertz linewidth and more than $10 \mathrm{~W}$ average power," Nat. Photon. 2, 55 (2008) and references therein.

[3] A. Chong, J. Buckley, W. Renninger, and F. W. Wise, “All-normal-dispersion femtosecond fiber laser," Opt. Exp. 14, 10095 (2006).

[4] O. Prochnow, R. Paschotta, E. Benkler, U. Morgner, J. Neumann, D. Wandt, and D. Kracht, "Quantum-limited noise performance of a femtosecond all-fiber ytterbium laser," Opt. Express 17, 15525 (2009).

[5] P. K. Mukhopadhyay, K. Özgören, I. L. Budunoglu, and F. Ö. Ilday, "All-fiber low-noise high-power femtosecond Yb-fiber amplifier system seeded by an all-normal dispersion fiber oscillator,” IEEE J. Sel. Top. Quantum Electron. 15, 145 (2009). 